\title{
Comparison of neurocognitive changes among newly diagnosed tuberculosis patients with and without dysglycaemia
}

Ernest Yorke ${ }^{1 *}$, Vincent Boima ${ }^{1}$, Ida Dzifa Dey ${ }^{1}$, Vincent Ganu², Norah Nkornu ${ }^{3}$, Kelvin Samuel Acquaye ${ }^{4}$ and C. Charles Mate-Kole $3,5,6$

\begin{abstract}
Background: Diabetes often occurs together with tuberculosis (TB) and both may affect each other negatively. Diabetes may be associated with neurocognitive dysfunctioning in affected patients and may negatively impact treatment adherence and outcomes. This study compared the neurocognitive status between newly diagnosed smear positive tuberculosis patients with dysglycaemia and those with normoglycaemia.
\end{abstract}

Methods: The current study was a cross-sectional study involving one hundred and forty-six (146) newly diagnosed smear positive TB patients. Oral glucose tolerance test (OGTT) was performed and the results were categorized as either normoglycaemia, impaired glucose tolerance (IGT), impaired fasting glucose (IFG) or diabetes.

Neurocognitive functioning among study participants was assessed at the time of TB diagnosis using Cognitive Failure Questionnaire (CFQ), Montreal Cognitive Assessment tool (MoCA), California Verbal Learning Test (CVLT), Brief Symptom Inventory (BSI) and the Spitzer Quality of Life Index (QLI).

Results: The mean age of the participants $(n=146)$ was 38.7 years with $78.8 \%$ being males and $21.2 \%$ females. Using the fasting blood glucose test, the prevalence of impaired fasting glucose and diabetes were 5.5 and $3.4 \%$ respectively, both representing a total of 13 out of the 146 participants; whilst the prevalence of impaired glucose tolerance and diabetes using 2-h post-glucose values were 28.8 and $11.6 \%$ respectively, both representing a total of 59 out of the 146 participants.

There were no significant differences in the mean scores on the neurocognitive measures between the dysglaycaemia and normoglycamic groups using fasting plasma glucose (FPG). However, there were significant differences in the mean scores between the dysglycaemia and normal groups using 2-h postprandial (2HPP) glucose values on Phobic Anxiety (Normal, Mean $=0.38 \pm 0.603$; dysglycaemia, Mean $=0.23 \pm 0.356 ; p=0.045$ ), and Montreal Cognitive Assessment (MoCA) scores (17.26 \pm 5.981 vs. $15.04 \pm 5.834, p=0.037$ ).

Conclusion: Newly diagnosed smear positive patients with dysglycaemia were associated with significantly lower mean cognitive scores and scores on phobic anxiety than those with normoglyacaemia. The latter finding must be further explored.

Keywords: Tuberculosis, Smear positive, Dysglycaemia, Neuropsychological disorders

\footnotetext{
* Correspondence: pavlovium@yahoo.com

'Department of Medicine \& Therapeutics, School of Medicine \& Dentistry, College of Health Sciences, University of Ghana, Legon, Accra, Ghana

Full list of author information is available at the end of the article
}

C C The Author(s). 2020 Open Access This article is licensed under a Creative Commons Attribution 4.0 International License, which permits use, sharing, adaptation, distribution and reproduction in any medium or format, as long as you give appropriate credit to the original author(s) and the source, provide a link to the Creative Commons licence, and indicate if changes were made. The images or other third party material in this article are included in the article's Creative Commons licence, unless indicated otherwise in a credit line to the material. If material is not included in the article's Creative Commons licence and your intended use is not permitted by statutory regulation or exceeds the permitted use, you will need to obtain permission directly from the copyright holder. To view a copy of this licence, visit http://creativecommons.org/licenses/by/4.0/ The Creative Commons Public Domain Dedication waiver (http://creativecommons.org/publicdomain/zero/1.0/) applies to the data made available in this article, unless otherwise stated in a credit line to the data. 


\section{Background}

A lot of effort continues to be made to reduce the worldwide incidence, morbidity and mortality associated with tuberculosis (TB); however it continues to be a deadly communicable disease. In 2015, 10.4 million new cases and 1.4 million deaths due to $\mathrm{TB}$ were estimated to have occurred by World Health Organisation (WHO) [1]. Many reviews and meta-analysis have reported that diabetes increases TB risk by between 1.5 to 7.8 fold $[2,3]$. Since developing countries have the greatest burden of $\mathrm{TB}$, the expected increase in the prevalence of type 2 diabetes in these parts of the world would increase the impact of diabetes on TB $[1,4]$. Negative impact of comorbid diabetes on TB include poor treatment outcomes [2], more severe disease and re-activation of dormant tuberculosis foci [5].

Tuberculosis (TB) like many chronic diseases is associated with a high burden of psychological disorders which may have a negative impact on the psychological health, treatment adherence and outcomes [6].

Common psychological disorders among TB patients especially around the time of diagnosis include depression, anxiety, in addition to poor quality of life [6]. The reasons for these changes include physiological and physical impact of the disease, stigma and isolation, effects of medications and rigours of keeping appointment and other negative social impact including loss of income [6]. These problems may affect self-esteem and attitudes towards disease management including compliance to medications and eventually lead to poor disease outcome [6]. The association of cognitive impairment with pulmonary TB alone does not appear to be strong, although this may be the case among patients with systemic spread [7-9]. However, cognitive dysfunction has been strongly associated with diabetes, which may impair self-management activities in many patients [10-12].

The negative impact of diabetes on TB, including potential cognitive impairment, coupled with the expected increases in the prevalence of both diseases is likely to portend bad prognosis in the management of TB patients [1, 2, 4-6]. Unfortunately, developing nations have limited healthcare budgets and resources to deal with the expected negative reciprocal impact of diabetes and TB on the affected population.

Despite the plethora of information on the potential interaction and effect of diabetes on cognitive function in general population, there is minimal published data about the potential cognitive impact of diabetes and dysglycaemia on cognition among TB patients $[2,3,5,6]$. This study examined and compared the neurocognitive functioning among newly diagnosed smear positive tuberculosis patients at time of diagnosis with dysglycaemia and those without.

\section{Methods}

\section{Study site and design}

We conducted a hospital based cross-sectional study at the Chest Clinic of the Korle-Bu Teaching Hospital, which is a tertiary care facility and the largest hospital in Ghana. The Korle-Bu Chest clinic is the main referral centre in southern Ghana for TB cases and other respiratory conditions.

\section{Participants and recruitment}

Inclusion criteria included TB patients who were newly diagnosed smear positive cases and were either initially diagnosed or referred for treatment at the Chest Clinic, 18 years and above with no previous history of TB treatment. None of the patients had known pre-existing diabetes. Excluded participants were those aged below 18 years, those with previous history of TB treatment, patients with smear negative TB, extra pulmonary TB, those with previous history of psychological, psychiatric or neurological disorders or those who refused consent. We used a convenient sampling method to recruit about 3-4 participants on a weekly basis (week days only) who met the included criteria. A total of 160 participants were recruited over a 12-month period.

\section{Measurements}

At enrolment, data on demographic and anthropometric characteristics such as body mass index (BMI), waist circumference (WC), hip circumference (HC) and waisthip-ratio (WHR) as well as medical history were collected using standardized questionnaire.

BMI (calculated) was categorized as obese, overweight, normal and underweight with defined values of 30.0 or more, 25-29.9, 18.5-24.9-and less than $18.5\left(\mathrm{Kg} / \mathrm{m}^{2}\right)$ respectively [13].

Microscopy using Ziehl-Neelsen (ZN) stain was used to determine the presence of acid fact bacilli (AFBs) [14]; whilst plasma glucose levels were determined using a $75 \mathrm{~g}$ oral glucose tolerance test (OGTT). After an overnight fast $(8 \mathrm{~h})$, ten millilitres $(\mathrm{ml})$ of fasting blood sample was taken into fluoridated blood sample tubes and centrifuged within 15 min of blood draw after being kept on ice [15]. After $2 \mathrm{~h}$ of the administration of $75 \mathrm{~g}$ glucose in $250 \mathrm{ml}$ water a second blood sample was taken into fluoride tubes for glucose determination. A commercial glucose oxidase reagent kits and controls (Diasys, GmBH, Germany) was used to determine plasma glucose; which were then categorized into normal glucose levels, impaired glucose tolerance (IGT), impaired fasting glucose (IFG) and diabetes.

A FPG and 2HPP values of $6.1-6.9 \mathrm{mmol} / \mathrm{l}$ and $7.8-$ $11 \mathrm{mmol} / \mathrm{l}$ diagnosed impaired fasting and glucose tolerance respectively; whilst FPG and $2 \mathrm{HPP}$ are $>7 \mathrm{mmol} / \mathrm{l}$ and $>11.1 \mathrm{mmol} / \mathrm{l}$ respectively or a person on regular 
medication for diabetes was labelled a diabetic. Normal plasma glucose values were defined by FPG and $2 \mathrm{HPP}$ values below $6.1 \mathrm{mmol} / \mathrm{l}$ and $7.8 \mathrm{mmol} / \mathrm{l}$ respectively [16]. Using fasting blood glucose values, dysglycaemia was defined as those with IFG and/or diabetes whilst using 2HPP values, it was defined as those with IGT and/or diabetes. All patients diagnosed with any form of dysglycaemia were referred to diabetes specialists for continued care [16].

Neuropsychological measurement tools include the Cognitive Failure Questionnaire (CFQ) [17], Montreal Cognitive Assessment tool (MoCA) [18], California Verbal Learning Test (CVLT) [19], Brief Symptom Inventory (BSI) [20] and the Spitzer Quality of Life Index (QLI) [21]. BSI assesses various psychological domains including depression, anxiety, somatization, paranoid ideation, phobia anxiety, obsessive compulsive behaviour, and interpersonal sensitivity [20]. For the purposes of this study only depression, anxiety, somatization, phobia anxiety as well as the Global severity index (GSI) of the BSI [22] were analysed. GSI of the BSI is calculated using the sums for the nine symptom dimensions plus the four additional items not included in any of the dimension scores, and dividing by the total number of items to which the individual responded. If no items were skipped the GSI will be the mean for all 53 items. The mean scores of the various domains including GSI are interpreted by comparison to age-appropriate norms [20]. The Spitzer Quality of Life Index (QLI) [21] rates patient's well-being in the areas of health, activity, daily living, outlook and support. The QLI yields a score that ranges from a high of 10 to a low of 0 . The higher one's score on this measure, the better his or her quality of life. Cognitive Failure Questionnaire (CFQ) [17] assesses self-reported cognitive lapses in perception, memory and misdirected action. The total score on the scale is obtained by adding up the ratings of the 25 individual items, yielding a score from 0 to 100. Score ranges between 0 and 50 is within the average scores and reflect normal functioning. Scores above 50 reflects clinically relevant problems. Montreal Cognitive Assessment tool (MoCA) [18] is a widely used screening assessment for detecting cognitive impairment. MoCA scores range between 0 and 30. A score of 26 or over is considered to be normal.

CVLT is a measure of verbal learning and memory, which demonstrates sensitivity to a range of clinical conditions such as recall, cognition and encoding [19]. CVLT short form was used; It consists 9 items and there are no cut-offs for normality, however the higher the scores (the number of items one can recall) the better the memory.

\section{Statistical analysis}

The Statistical software Stata version 15 was used for the analysis of data of 146 patients with complete OGTT information. It was imported from Microsoft Excel version 2010 following an initial cleaning.

Socio-demographic, anthropometric, medical history and glycaemic variables were summarised descriptively as means and standard deviations, frequencies and percentages, For the purposes of analysis, dysglycaemia (abnormal glucose level) was defined to include the combined prevalence of impaired fasting glucose and diabetes (using fasting glucose values) or those with impaired glucose tolerance and diabetes (using 2-h postprandial values). Mean baseline scores of participants on neurocognitive measures tools were compared using an independent $\mathrm{T}$-test at baseline between those with dysglycaemia and those without using both FBS and 2HPP values. The statistical significance levels were set at $5 \%$.

\section{Results}

One hundred and forty-six (146) participants (78.8\% males and $21.2 \%$ females) with a mean age of 38.7 years were involved in the study. Majority $(51.37 \%)$ of our participants were single with $36.99 \%$ having education up to Junior High School (JHS) level (Table 1). Fasting blood glucose tests results showed $5.48 \%$ had impaired glucose levels whilst $3.42 \%$ had diabetes (Table 1 ). 2-h postprandial results showed $28.77 \%$ had impaired glucose levels whilst $11.64 \%$ had diabetes (Table 1).

Information on the prevalence of diabetes and dysglycaemia in this study shown in Table 1 has been published earlier and discussed extensively [23].

\section{Mean scores on neuropsychological testing}

The independent t-test conducted to compare the differences in the scores on neurocognitive tests (CFQ, MOCA, and CVLT) between dysglycaemia and those with normal glucose, using FBS showed no significant differences between the two groups.

However, on the behavioural measures there were significant differences between those with dysglycaemia and those without using $2 \mathrm{HPP}$ scores, and the scores of Phobic Anxiety $\mathrm{t}(150)=2.025, p=0.045$. Participants' with dysglycaemia had lower scores compared to those who had normal glucose values (Normal, Mean $=0.38 \pm 0.603 ;$ dysglycaemia, Mean $=$ $0.23 \pm 0.356)$, Table 2 .

The scores on the Montreal Cognitive Assessment (MOCA) tool showed a significant difference in the mean scores between those with normal vs. dysglycaemia, $\mathrm{t}(105)=2.112, p=0.037$. Those with dysglycaemia had lower mean scores (Mean $=15.04 \pm 5.834$ ) compared with those with normal glucose (Mean $=17.26 \pm 5.981$ ). There were no significant differences in the rest of the mean scores for the other tests (Table 2). 
Table 1 Background Characteristics of newly diagnosed smear positive tuberculosis patients at the Chest Clinic of the Korle-Bu Teaching hospital in Accra, Ghana, 2017

\begin{tabular}{|c|c|c|}
\hline & Frequency & Percentage \\
\hline Age (mean $\pm S D)$ & $38.70 \pm 13.97$ & \\
\hline \multicolumn{3}{|l|}{ Sex } \\
\hline Female & 31 & 21.20 \\
\hline Male & 115 & 78.80 \\
\hline \multicolumn{3}{|l|}{ Highest educational level } \\
\hline Tertiary & 23 & 15.75 \\
\hline O-Level/A-level/SHS & 32 & 21.92 \\
\hline Middle School/ JHS & 54 & 36.99 \\
\hline Primary & 14 & 9.59 \\
\hline None & 13 & 8.90 \\
\hline Other & 10 & 6.85 \\
\hline \multicolumn{3}{|l|}{ Marital Status } \\
\hline Single & 75 & 51.37 \\
\hline Married & 62 & 42.47 \\
\hline Separated/Divorced & 9 & 6.16 \\
\hline \multicolumn{3}{|l|}{ Employment status } \\
\hline Unemployed & 38 & 26.03 \\
\hline Employed & 108 & 73.97 \\
\hline \multicolumn{3}{|l|}{ Alcohol intake } \\
\hline Yes & 22 & 15.07 \\
\hline No & 124 & 84.93 \\
\hline \multicolumn{3}{|l|}{ Smoking } \\
\hline Yes & 10 & 6.85 \\
\hline No & 136 & 93.15 \\
\hline Weight (mean \pm SD) & $52.74 \pm 8.94$ & \\
\hline Height (mean \pm SD) & $1.68 \pm 0.08$ & \\
\hline \multicolumn{3}{|l|}{ BMI } \\
\hline Above 30 (Obese) & 1 & 0.68 \\
\hline 25-29.9 (Overweight) & 2 & 1.37 \\
\hline 18.5-24.9 (Normal) & 40 & 27.4 \\
\hline Below 18.5 (Underweight) & 103 & 70.55 \\
\hline Mean \pm SD & $18.49 \pm 3.00$ & \\
\hline \multicolumn{3}{|l|}{ 2-HPP Glucose (mmol/L) } \\
\hline Diabetes (> 11.1) & 17 & 11.64 \\
\hline Impaired (7.8-11.1) & 42 & 28.77 \\
\hline Normal $(<7.8)$ & 87 & 59.59 \\
\hline Mean \pm SD & $8.35 \pm 3.44$ & \\
\hline \multicolumn{3}{|c|}{ Fasting Plasma Glucose (mmol/L) } \\
\hline Diabetes $(>7.1)$ & 5 & 3.42 \\
\hline Impaired (6.1-7) & 8 & 5.48 \\
\hline Normal $(<6.1)$ & 133 & 91.1 \\
\hline Mean \pm SD & $5.12 \pm 1.52$ & \\
\hline
\end{tabular}

SD Standard deviation, DBP Diastolic Blood pressure, SBP Systolic Blood Pressure, 2HPP 2-h postprandial

\section{Discussion}

In this study, using fasting glucose values, the prevalence of impaired fasting glucose and diabetes were 5.5 and $3.4 \%$ respectively. Using 2 -h post-glucose, the prevalence of impaired glucose tolerance and diabetes were 28.8 and $11.6 \%$ respectively. These prevalence findings have already been published and discussed extensively [23] with similar results compared to other studies [24-26]. Significantly, most of the patients (about 70\%) were underweight. TB patients tend to come from low socioeconomic background and generally malnourished and underweight [27]. Weight loss among TB patients may also be contributed to by the inflammatory response to infection $[28,29]$ as well as nausea, loss of appetite and vomiting.

\section{Diabetes and cognition decline among TB patients}

The 2-h post-glucose values revealed that $\mathrm{TB}$ patients with dysglycaemia had a significant lower mean score than those with normal glucose values on MOCA. This implies that dysglycaemia is associated with poorer cognitive functioning compared to those with normal glucose levels among TB patients. This finding supports the conclusions from other studies that diabetes predisposes to cognitive decline both in humans and animal models [12, 30-33], and this finding also holds true for our study cohort who had TB and dysglycaemia. Cognitive decline may negatively affect compliance to the strict requirements of TB treatment including adherence to medications which can potentially worsen disease outcomes [34].

Whiles the pathophysiology of cognitive dysfunction in diabetes mellitus is putative; hyperglycaemia, vascular disease, hypoglycaemia, and insulin resistance are thought to play important roles [35]. Altered cerebral insulin and glucose homoeostasis may lead to widespread brain microangiopathy $[36,37]$. Insulin resistance, a feature of diabetes and prediabetes, increases the formation of Advanced Glycation End-products (AGE) leading to the overproduction of Reactive Oxygen Species (ROS) which accelerate biological aging and subsequent cognitive decline [38]. The recognition of cognitive impairment in the dysglycaemia group in a relatively younger patient cohort in our study (average age 38.7 years) might suggest that cognitive impairment that occurs in patients with dysglycaemia and diabetes in TB patients probably occurs early on in the disease. This was supported by a study by Ruis et al. who found out that early on in the course of type 2 diabetes, there is modest cognitive decline [39].

The influence of pulmonary TB alone on cognitive decline does not appear to be strong. A study published in India in 2015 showed that cognitive impairment was significantly higher among HIV patients with Pulmonary 
Table 2 Summary of Independent t-test for 2HPP and neuropsychological measures among newly diagnosed smear positive tuberculosis patients at the Chest Clinic of the Korle-Bu Teaching hospital in Accra, Ghana, 2017

\begin{tabular}{|c|c|c|c|c|c|c|c|}
\hline & $2 \mathrm{HPP}$ & $n$ & M & SD & $\mathrm{t}$ & df & $p$ \\
\hline \multirow[t]{2}{*}{ Cognitive Failures (CFQ) } & Normal & 93 & 19.23 & 13.20 & 1.25 & 152 & .202 \\
\hline & Abnormal & 61 & 16.61 & 11.86 & & & \\
\hline \multirow[t]{2}{*}{ Somatisation } & Normal & 92 & 1.27 & .81 & -.38 & 152 & .704 \\
\hline & Abnormal & 62 & 1.32 & .77 & & & \\
\hline \multirow[t]{2}{*}{ Anxiety } & Normal & 92 & .55 & .66 & 1.53 & 152 & .106 \\
\hline & Abnormal & 62 & .41 & .48 & & & \\
\hline \multirow[t]{2}{*}{ Depression } & Normal & 91 & .52 & .59 & .68 & 151 & .477 \\
\hline & Abnormal & 62 & .46 & .45 & & & \\
\hline \multirow[t]{2}{*}{ Phobic Anxiety } & Normal & 92 & .38 & .60 & 2.03 & 149.7 & .045 \\
\hline & Abnormal & 62 & .23 & .36 & & & \\
\hline \multirow[t]{2}{*}{ Perceived Psychological Distress (BSI-GSI) } & Normal & 92 & .65 & .47 & 1.21 & 151.8 & .229 \\
\hline & Abnormal & 62 & .58 & .30 & & & \\
\hline \multirow[t]{2}{*}{ Total Free Recall } & Normal & 70 & 20.13 & 5.83 & .87 & 105 & .353 \\
\hline & Abnormal & 37 & 19.16 & 4.65 & & & \\
\hline \multirow[t]{2}{*}{ Short Delay Free Recall } & Normal & 70 & 5.47 & 1.96 & 1.61 & 105 & .120 \\
\hline & Abnormal & 37 & 2.12 & 1.61 & & & \\
\hline \multirow[t]{2}{*}{ Long Delay Free Recall } & Normal & 68 & 4.76 & 2.02 & 1.03 & 102 & .307 \\
\hline & Abnormal & 36 & 4.33 & 2.04 & & & \\
\hline \multirow[t]{2}{*}{ Cognitive Impairment (MOCA) } & Normal & 84 & 17.26 & 5.98 & 2.10 & 132 & .037 \\
\hline & Abnormal & 50 & 15.04 & 5.83 & & & \\
\hline \multirow[t]{2}{*}{ Quality of Life } & Normal & 93 & 7.20 & 1.90 & 1.30 & 153 & .197 \\
\hline & Abnormal & 62 & 6.82 & 1.59 & & & \\
\hline
\end{tabular}

$p<.05$ (2-tailed), Note: M Mean, SD Standard deviation, $d f$ Degree of freedom, BSI Brief Symptom Inventory, GSI Global severity index, MOCA Montreal Cognitive Assessment, Abnormal Dysglyacaemia

Tuberculosis than in patients with HIV infection alone [40]. In the setting of non-clinically obvious systemic spread, especially among HIV patients, PTB may impact on neurocognitive function $[41,42]$ through granulomatous meningeal inflammation with the formation of exudate and adhesions, obliterative vasculitis, encephalitis or myelitis or hydrocephalus [7-9].

\section{Prediabetes and cognitive decline}

The dysglycaemia group included TB patients with prediabetes who scored significant lower scores on cognitive tests. In a recent study published in 2017 [43], prediabetes was associated with lower performance in memory in middle age and but a less steep decline in memory over the follow-up period [43]. Also, the Confucius Hometown Aging Project, which was a cross-sectional study of 1528 participants conducted in Shandong province in China, examined the association of diabetes and prediabetes with cognitive impairment and depression among Chinese elderly people aged $\geq 60$ years. Cognitive impairment and depression was found in 24.7 and $20.3 \%$ subjects respectively. The multiple adjusted odds ratio (OR) of cognitive impairment was 1.61 for prediabetes, 1.38 for diabetes, and 1.43 for having either prediabetes or diabetes [44]. .Another study found modest cognitive decline already present at the early stage of type 2 diabetes [39]. The results of this study are preliminary and our future study will employ more neuropsychological measures and larger numbers to assess various domains of cognition, and compare any differences between those with overt diabetes and prediabetes.

\section{Phobic anxiety}

Our study revealed that participants with dysglycaemia had significantly lower scores on Phobic Anxiety compared to those who had normal glucose levels. This means that participants with dysglycemia reported less phobic anxiety compared to those with normal glucose levels. This finding however differs from other publications, which found higher anxiety related symptoms including phobic anxiety associated with diabetes [45-47]. Differences in participant characteristics such as the relatively young age of subjects and a different setting of the study may account for the observed differences in the findings. This must further be explored in subsequent studies. 


\section{Future research}

Future research must include extensive neuropsychological measures to assess various cognitive domains and establish the severity and degree of cognitive decline that may impact treatment outcomes among TB patients. Also since social, demographic and sanitary variables are often implicated in psychological issues concerning TB patients, these factors could be explored in the future studies as moderators/modulators of neurocognitive function as well.

\section{Conclusion}

Our study is unique in examining the influence of dysglyacaemia on cognitive performance among young adults with TB. Newly diagnosed smear positive TB patients with dysglycaemia was associated with statistically significantly lower mean cognitive scores than those with normal glucose values. Also, patients with abnormal glucose values was associated with statistically lower mean scores on phobic anxiety as compared to those who were normoglycaemic; and this finding must be further explored.

The study findings have implications TB management. Among newly diagnosed smear positive patients, neuropsychological tests must be performed at diagnosis to ascertain the presence or otherwise of any neuropsychological deficits. Any deficits identified should be promptly treated to reduce its impact on the patient as well to improve treatment outcomes. It is hoped that this will spur increased interest and research on the impact of dysglycaemia on the cognitive function on TB patients and its impact on treatment outcomes.

\section{Abbreviations}

AFB: Acid fact bacilli; BMI: Body mass index; BP: Blood pressure; BSI: Brief Symptom Inventory; CFQ: Cognitive Failure Questionnaire; Cl: Confidence Interval; CVLT: California Verbal Learning Test; FPG: Fasting plasma glucose; IGT: Impaired glucose tolerance; MDR: Multidrug resistant tuberculosis; MOCA: Montreal Cognitive Assessment tool; OGTT: Oral glucose tolerance test; OR: Odds ratio; SD: Standard deviation; QLI: Spitzer Quality of Life Index; TB: Tuberculosis; WHO: World Health Organisation; ZN: Zeihl Neelson; 2HPP: 2-Hour Post-prandial glucose

\section{Acknowledgments}

We also appreciate the contribution of Ama Aidoo and Ernest AmaningKwarteng especially with respect to data collection.

\section{Authors' contributions}

EY conceived the study, participated in its design, data collection, analysis, drafted the manuscript and collation of all drafts. VB, IDD, VG, NN, KSA and CCM contributed to study design, data collection, analysis and manuscript draft. All authors read and approved the final version of the manuscript.

\section{Funding}

This study was mostly funded by a University of Ghana Office of Research, Innovation and Development (ORID) grant (Project Reference Number: URF/ 9/ILG-076/2015-2016). The funds supported the design of the study and collection, analysis, and interpretation of data.

\section{Availability of data and materials}

The datasets used and/or analysed during the current study are available from the corresponding author on reasonable request.

\section{Ethics approval and consent to participate}

All patients provided written informed consent. Ethical and Protocol approval for the study was sought from the College of Health Sciences Ethics and Protocol Review Committee of the University of Ghana with reference number URF/9/ILG-076/2015-2016. It complied with the Helsinki Declaration of 1964 (Revised 2013) on human experimentation. Strict confidentiality of data and privacy for study participants were ensured. Data was kept secured and was available only to the principal investigator. Patients found to have dysglycaemia and/or psychological disorders were referred for further assessment and possible treatment.

Consent for publication

Not applicable.

\section{Competing interests}

The authors declare that they have no competing interests.

\section{Author details}

${ }^{1}$ Department of Medicine \& Therapeutics, School of Medicine \& Dentistry, College of Health Sciences, University of Ghana, Legon, Accra, Ghana. ${ }^{2}$ Department of Medicine, Korle-Bu Teaching Hospital, Accra, Ghana. ${ }^{3}$ Department of Psychology, School of Social Sciences, College of Humanities, University of Ghana, Accra, Ghana. ${ }^{4}$ Department of Social and Behavioural Sciences, School of Public Health, University of Ghana, Accra, Ghana. ${ }^{5}$ Department of Psychiatry, School of Medicine \& Dentistry, College of Health Sciences, Korle-Bu, Accra, Ghana. ${ }^{6}$ Centre for Ageing Studies, College of Humanities, University of Ghana, Accra, Ghana.

Received: 11 April 2019 Accepted: 25 March 2020

Published online: 03 April 2020

\section{References}

1. World Health O: Global tuberculosis report 2016. 2016.

2. Stevenson CR, Critchley JA, Forouhi NG, Roglic G, Williams BG, Dye C, Unwin NC. Diabetes and the risk of tuberculosis: a neglected threat to public health? Chronic IIIn. 2007;3(3):228-45.

3. Jeon CY, Murray MB. Diabetes mellitus increases the risk of active tuberculosis: a systematic review of 13 observational studies. PLoS Med. 2008;5(7):0050152

4. International Diabetes Federation. IDF Diabetes Atlas, 8th edn. Brussels: International Diabetes Federation; 2017. Available at www.idf.org/e-library/ epidemiology-research/diabetes-atlas/134-idf-diabetes-atlas-8th-edition.html. Accessed 9 July 2019.

5. Wilson RM. Infection and diabetes mellitus. In: Pickup JC, Williams G, editors. Textbook of diabetes. Oxford: Blackwell Scientific Publication; 1991. p. 813-9.

6. Peltzer K, Naidoo P, Matseke G, Louw J, McHunu G, Tutshana B. Prevalence of psychological distress and associated factors in tuberculosis patients in public primary care clinics in South Africa. BMC Psychiatry. 2012;12(89):12-89.

7. Grant I, Marcotte TD, Heaton RK, Group H. Neurocognitive complications of HIV disease. Psychol Sci. 1999;10(3):191-5.

8. Garg RK. Tuberculous meningitis. Acta Neurol Scand. 2010;122(2):75-90.

9. Chen H-L, Lu C-H, Chang C-D, Chen P-C, Chen M-H, Hsu N-W, Chou K-H, Lin W-M, Lin C-P, Lin W-C. Structural deficits and cognitive impairment in tuberculous meningitis. BMC Infect Dis. 2015;15(1):279.

10. Munshi MN. Cognitive dysfunction in older adults with diabetes: what a clinician needs to know. Diabetes Care. 2017;40(4):461-7.

11. Ott A, Stolk RP, Van Harskamp F, Pols HAP, Hofman A, Breteler MMB. Diabetes mellitus and the risk of dementia the Rotterdam study. Neurology. 1999;53(9):1937.

12. Biessels GJ, Staekenborg S, Brunner E, Brayne C, Scheltens P. Risk of dementia in diabetes mellitus: a systematic review. Lancet Neurol. 2006;5(1): 64-74.

13. Alberti G, Zimmet P, Shaw J, Grundy SM. The IDF consensus worldwide definition of the metabolic syndrome. Brussels: Int Diabetes Federation. 2006;23(5):469-80 
14. Eisenach K, Siddiqi S: WHO Mycobacteriology laboratory manual. Available at https://www.who.int/tb/laboratory/mycobacteriology-laboratory-manual. pdf. Accessed 9 July 2019.

15. Sacks DB, Bruns DF, Goldstein DE, et al. Guidelines and recommendations for laboratory analysis in the diagnosis and management of diabetes mellitus. Clin Chem. 2002;48(3):436-72.

16. World Health Organization: International Diabetes Federation (2006) Definition and diagnosis of diabetes mellitus and intermediate hyperglycemia: report of a WHO/IDF consultation. IDF consultation 2008.

17. Broadbent DE, Cooper PF, FitzGerald P, Parkes KR. The cognitive failures questionnaire (CFQ) and its correlates. Br J Clin Psychol. 1982;21(Pt 1):1-16.

18. Nasreddine ZS, Phillips NA, Bédirian V, Charbonneau S, Whitehead V, Collin I, Cummings JL, Chertkow H. The Montreal cognitive assessment, MoCA: a brief screening tool for mild cognitive impairment. J Am Geriatr Soc. 2005; 53(4):695-9.

19. Elwood RW. The California verbal learning test: psychometric characteristics and clinical application. Neuropsychol Rev. 1995;5(3):173-201.

20. Derogatis LR. BSI, brief symptom inventory: administration, scoring \& procedures manual: National Computer Systems; 1993.

21. Spitzer WO, Dobson AJ, Hall J. Measuring the quality of life of cancer patients. J Chron Dis. 1981;34:595.

22. Derogatis LR, Savitz KL. The SCL-90-R and brief symptom inventory (BSI) in primary care; 2000

23. Yorke E, Boima V, Dey ID, Atiase Y, Akpalu J, Yawson AE, Ganu V, Forson A, Mate-Kole CC. Examination of Dysglycaemia among newly diagnosed tuberculosis patients in Ghana: a cross-sectional study. Tuberc Res Treat. 2018;2018:1830372.

24. Ponce-de-Leon A, de Lourdes G-GM, Garcia-Sancho MC, Gomez-Perez FJ, Valdespino-Gomez JL, Olaiz-Fernandez G, Rojas R, Ferreyra-Reyes L, CanoArellano B, Bobadilla M. Tuberculosis and diabetes in southern Mexico. Diabetes Care. 2004;27(7):1584-90.

25. Pablos-Mendez A, Blustein J, Knirsch CA. The role of diabetes mellitus in the higher prevalence of tuberculosis among Hispanics. Am J Public Health. 1997:87(4):574-9.

26. Singla R, Khan N, Al-Sharif N, Al-Sayegh MO, Shaikh MA, Osman MM. Influence of diabetes on manifestations and treatment outcome of pulmonary TB patients. Int J Tuberc Lung Dis. 2006;10(1):74-9.

27. Oxlade O, Murray M. Tuberculosis and poverty: why are the poor at greater risk in India? PLoS One. 2012;7(11):e47533.

28. Matthys P, Billiau A. Cytokines and cachexia. Nutrition. 1997;13(9):763-70.

29. van Crevel $R$, Karyadi $E$, Netea MG, Verhoef $H$, Nelwan $R H H$, West $C E$, van der Meer JWM. Decreased plasma leptin concentrations in tuberculosis patients are associated with wasting and inflammation. J Clin Endocrinol Metab. 2002;87(2):758-63.

30. Wong RHX, Scholey A, Howe PRC. Assessing premorbid cognitive ability in adults with type 2 diabetes mellitus - a review with implications for future intervention studies. Curr Diab Rep. 2014;14(11):547.

31. Monette MCE, Baird A, Jackson DL. A meta-analysis of cognitive functioning in nondemented adults with type 2 diabetes mellitus. Can J Diabetes. 2014; 38(6):401-8

32. Palta P, Schneider ALC, Biessels GJ, Touradji P, Hill-Briggs F. Magnitude of cognitive dysfunction in adults with type 2 diabetes: a meta-analysis of six cognitive domains and the most frequently reported neuropsychological tests within domains. J Int Neuropsychol Soc. 2014;20(3):278-91.

33. Grünblatt $E$, Bartl J, Riederer $P$. The link between iron, metabolic syndrome, and Alzheimer's disease. J Neural Transm. 2011;118(3):371-9.

34. Rohde D, Merriman NA, Doyle F, Bennett K, Williams D, Hickey A. Does cognitive impairment impact adherence? A systematic review and metaanalysis of the association between cognitive impairment and medication non-adherence in stroke. PLoS One. 2017;12(12):e0189339.

35. Kodl CT, Seaquist ER. Cognitive dysfunction and diabetes mellitus. Endocr Rev. 2008;29(4):494-511.

36. Gasparini $L, X u H$. Potential roles of insulin and IGF-1 in Alzheimer's disease. Trends Neurosci. 2003:26(8):404-6.

37. Craft S, Watson GS. Insulin and neurodegenerative disease: shared and specific mechanisms. Lancet Neurol. 2004;3(3):169-78.

38. Smith MA, Sayre LM, Monnier VM, Perry G. Radical AGEing in Alzheimer's disease. Trends Neurosci. 1995:18(4):172-6.

39. Ruis C, Biessels GJ, Gorter KJ, Van Den Donk M, Kappelle LJ, Rutten GEHM. Cognition in the early stage of type 2 diabetes. Diabetes Care. 2009;32(7): $1261-5$.
40. Saad MZ, Rao ER, Archana V. A Study of Cognitive Impairment in HIV-TB CoInfection. J Dental Med Sci. 2015;14(8 Version VIII):10-3.

41. Chinyama J, Ngoma MS, Menon AJ, Hestad K, Heaton RK. The effect of pulmonary tuberculosis on neurocognitive function in HIV infected adult patients in Lusaka, Zambia. Med J Zambia. 2016;43(4):199-206.

42. Thomas TY, Rajagopalan S. Tuberculosis and aging: a global health problem. Clin Infect Dis. 2001;33(7):1034-9.

43. Marseglia A, Dahl Aslan AK, Fratiglioni L, Santoni G, Pedersen NL, Xu W. Cognitive trajectories of older adults with Prediabetes and diabetes: a population-based cohort study. J Gerontol. 2017;73(3):400-6.

44. Yan Z, Cai C, Song H, Jiang H, Sun B, Bai B, Qiu C. Association of diabetes and prediabetes with cognitive impairment and depression among Chinese elderly people: the Confucius hometown aging project. Alzheimer's \& Dementia: J Alzheimer's Assoc. 2012;8(4):313

45. Grigsby AB, Anderson RJ, Freedland KE, Clouse RE, Lustman PJ. Prevalence of anxiety in adults with diabetes: a systematic review. Diabetes. 2001;50: A540.

46. Lustman PJ. Anxiety disorders in adults with diabetes mellitus. Psychiatr Clin. 1988;11(2):419-32.

47. Farvid MS, Qi L, Hu FB, Kawachi I, Okereke Ol, Kubzansky LD, Willett WC. Phobic anxiety symptom scores and incidence of type 2 diabetes in US men and women. Brain Behav Immun. 2014:36:176-82.

\section{Publisher's Note}

Springer Nature remains neutral with regard to jurisdictional claims in published maps and institutional affiliations.
Ready to submit your research? Choose BMC and benefit from:

- fast, convenient online submission

- thorough peer review by experienced researchers in your field

- rapid publication on acceptance

- support for research data, including large and complex data types

- gold Open Access which fosters wider collaboration and increased citations

- maximum visibility for your research: over $100 \mathrm{M}$ website views per year

At $\mathrm{BMC}$, research is always in progress.

Learn more biomedcentral.com/submissions 